\title{
Improving the quality of referrals to ambulatory emergency care
}

\author{
Authors: Katherine Keaney, Annelies Sweeney, Shaznin Visanji," Amber Hawksley* and Reginald Coleman
}

\section{Introduction}

In January 2019, Barnet Hospital opened a new acute medical unit (AMU), which uses the objectives and aims set out by NHS Improvement's Same day emergency care. ${ }^{1}$ The ambulatory emergency clinic (AEC) forms part of the new AMU, as a clinic for those patients 'fit to sit', to avoid unnecessary hospital admissions in clinically stable, ambulatory patients. The aim of this audit was to understand how the clinic was being utilised, prior to the new AMU opening. By identifying the most common reasons for referral and highlighting any unsuitable referrals, the community and medical teams were educated, therefore utilising the services better for on-the-day referrals.

\section{Materials and methods}

Baseline data was collected of all new referrals made to the AEC in 1 week. Data was collected on the source of each referral, the number of referrals that were accepted by the AEC consultant, the clinical reason for referral and any subsequent referrals to other clinics or specialties. A re-audit was completed 4 weeks after the new AMU opened.

\section{Results and discussion}

118 referrals to AEC were audited over a 1 -week period. $36 \%$ of referrals were from the 'acute medical' on-call team, $28 \%$ from the emergency department, $24 \%$ from GPs and $12 \%$ from inpatient wards (post discharge). $31 \%$ of all new referrals were rejected by the $A E C$ consultant, requiring discussion with the referring clinician or referral to an alternative service. The majority of 'inappropriate' referrals were from general practice, although $28 \%$ of inappropriate referrals were from the medical on-call team. The most common clinical presentations referred to AEC were suspected venous thromboembolism and infections other than cellulitis. $10 \%$ of all referrals were to repeat or follow-up blood tests. A total of $30 \%$ of all patients referred to AEC were later referred to other clinics and specialties, of which over half were sent to community clinics and to surgical specialties.

Authors: Barnet Hospital, Royal Free NHS Trust ${ }^{*} \mathrm{RCP}$ chief registrar
A re-audit was conducted 4 weeks after the new AMU opened, 88 patients were audited over a 1 -week period. Following a grand round presentation and internal communications to the medical teams, there was significant reduction in the percentage of rejected referrals (18\%), and onward referrals to other specialties (19\%). Although the percentage of patients seen in AEC post discharge from medical ward had gone up to $32 \% ; 75 \%$ of these patients were discharged from the acute medical wards, aiding in admission reduction and reduced length of stay. Unfortunately, there was an increase in the number of referrals to follow-up blood test results to $28 \%$.

\section{Conclusion}

The results from the audit reflect the large number of patients being referred to AEC inappropriately and the burden of medical and surgical subspecialties on this outpatient resource. We recommended revising the current guidelines to outline clear referral criteria. A pathway is currently in progress for access to 'Hot clinics' for medical teams to referral to directly. A protocol has been implemented for consultants to follow up test results requested on their on-call, including blood tests, to reduce the follow-up burden on the clinic.

\section{Reference}

1 NHS Improvement and the Ambulatory Care Network. Same day emergency care: clinical definition, patient selection and metrics. NHS Improvement, 2018:1-15. 